\title{
Security Evaluation of Distribution Network Based on Situation Awareness
}

\author{
Yanzhen $\mathrm{Li}^{1, \text { a }}$, Bo Peng ${ }^{1, \mathrm{~b}}$, Kaiqiang Cheng ${ }^{2, \mathrm{c}}$, Liangzi Li ${ }^{2, \mathrm{~d}}$, and Pengfei Shen ${ }^{2, \mathrm{e}}$ \\ ${ }^{1}$ State Grid Qingdao Power Supply Company, Qingdao 266002, China \\ ${ }^{2}$ School of electrical engineering, Shandong University, Jinan 250061, China

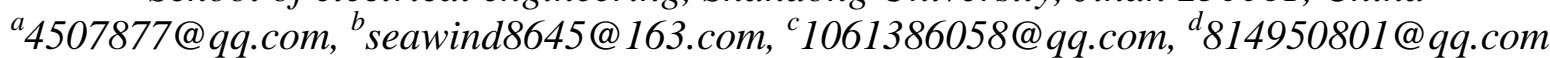 \\ '1477758543@qq.com
}

Keywords: Distribution Network, security, situation awareness

\begin{abstract}
With a large number of distributed sources connected to the distribution network, it brings challenges to the safe and stable operation of the distribution network, so it is very urgent to study the accurate and real-time evaluation of the distribution network security. This paper presents a security evaluation method for distribution network based on situation awareness. First of all, the indexes which can accurately evaluate the distribution network security are choosen. Then the state estimation is used to obtain a more accurate state quantity of distribution network in the stage of situation understanding. Then, the basic data needed for the evaluation process are obtained by power flow calculation, and the evaluation indexes are calculated. Finally, the grey prediction model is used to get the data of the future state in the stage of situation forcasting. The simulation results of IEEE14 bus show that the proposed method can reflect the security of distribution network in real time and accurately
\end{abstract}

\section{Introduction}

With photovoltaic generation, wind power and other new energy sources connected to the grid, it brings challenges to the safe operation of the distribution network. Therefore, monitoring, analysis and accurate evaluation of the distribution network operation status has become the focus of current research. Situation awareness (SA) has been applied in the fields of economy, computer, military and so on. It can accurately perceive the running state of the system, so we can use the technology of situation awareness to perceive the current state of distribution network to provide more reliable, comprehensive and accurate distribution network operation information for operators.

In [1], the frame, meaning and key technology of situation awareness in distribution network are put forward, and the necessary algorithms and mathematical models of the technology are not introduced in detail. In [2], the state estimation algorithm is used to process the current data, and the distribution network is sensed in real time, but without prediction. In [3], modeling based on historical data is provided, but the method of evaluating the security of distribution networks isn't introduced. In [4][5], the security index of distribution network is determined based on the topology structure of distribution network. These indexes are used for power network planning and the 
real-time performance is poor.

The above literature does not combine security evaluation with situational awareness, thus, the first part of this paper introduces some real-time index used to evaluate the security of the distribution network; the second part presents a method of situation awareness, including the state estimation based on weighted least square(WLS) method and the grey prediction; the third part introduces the simulation example, based on the whole process of situation awareness, the distribution network security evaluation is finished .

\section{Security Index of the Distribution Network}

This paper does not choose the security index from the aspect of the distribution network topology, but from the aspect of system running and considering the real time, the following indexes are screened out.

\subsection{Load Margin}

For the operators, they are usually concerned about the distance from the running crash point, so we can choose the current passing load rate to reflect the degree of stable operation of the system. The evaluation expression is as follows:

$$
\mathrm{SA}=\frac{\mathrm{P}_{\mathrm{cr}}-\mathrm{P}_{0}}{\mathrm{P}_{\mathrm{cr}}}
$$

$\mathrm{P}_{0}$ is the current load value of the system, $\mathrm{P}_{\mathrm{cr}}$ is the critical load value of the system. This index reflects the load pressure of the system.

\subsection{Voltage Margin}

There is strong coupling between voltage and reactive power in distribution system, and voltage margin reflects reactive power reserve ability of distribution network. The expression is:

$$
Q(V)=\left\{\begin{array}{cc}
0 & V \in\left(-\infty, V_{L}\right] \\
\frac{V-V_{L}}{V_{W L}-V_{L}} & V \in\left(V_{L}, V_{W L}\right] \\
1 & V \in\left(V_{W L}, V_{W H}\right] \\
\frac{V_{H}-V}{V_{H}-V_{W H}} & V \in\left(V_{W H}, V_{L}\right] \\
0 & V \in\left(V_{L},+\infty\right)
\end{array}\right.
$$

$\mathrm{V}$ is the actual voltage, $\mathrm{V}_{\mathrm{H}}$ is the maximum upper limit of voltage, $\mathrm{V}_{\mathrm{L}}$ is the maximum lower limit of voltage, $\mathrm{V}_{\mathrm{WH}}$ is the maximum upper warning value set, $\mathrm{V}_{\mathrm{WL}}$ is the maximum lower warning value set. This index reflects whether the voltage is stable in a safe range, and it can serve as a warning.

\subsection{Load Transfer Capacity}

Line load transfer capacity is reflected by line load transfer rate, the expression is:

$$
\eta_{1}=\frac{\mathrm{I}_{\mathrm{MAX} 2}-\mathrm{I}_{\mathrm{LM} 2}}{\mathrm{I}_{\mathrm{LM} 1}}
$$

$\mathrm{I}_{\text {MAX2 }}$ is receiving current limit, $\mathrm{I}_{\mathrm{LM} 2}$ is the receiving load current, $\mathrm{I}_{\mathrm{LM}}$ is the delivery load current. The larger the index is, the stronger the transferable capacity is. 


\subsection{Line Light Load Rate}

Line light load rate means that the actual current of the line is $20-50 \%$ of the rated current of the line. The expression of the line load is:

$$
\mathrm{r}_{\mathrm{LR}}=\frac{\mathrm{I}_{\mathrm{i}}}{\mathrm{I}_{\mathrm{L}}} \times 100 \%
$$

The expression of Line Light load rate is:

$$
r_{\mathrm{NLL}}=\frac{\mathrm{N}_{\mathrm{LL}}}{\mathrm{N}}
$$

$\mathrm{N}_{\mathrm{LL}}$ denotes the number of lines which is lightly loaded, $\mathrm{N}$ denotes the total number of lines, $\mathrm{I}_{\mathrm{i}}$ is actual current , $\mathrm{I}_{\mathrm{L}}$ is the rated current .

\section{SA of the Distribution Network}

SA of the distribution network goes through three stages. The first stage is acquiring data from some systems in the distribution network, including meteorological data, environmental data, measurement data, and so on. The second stage is comprehension .It is to comprehend the meaning or significance of the acquiring information in the distribution network by analyzing and processing data. The third stage is projection. It means that after the processing of the first two stages, we can analyze and summarize the development trend of the distribution network to predict what is likely to happen next. This paper uses a state estimation algorithm based on WLS as a means of situation comprehension and uses grey prediction algorithm as a means of situation projection.

\subsection{State Estimation Based On WLS}

State estimation is a key part of situation awareness in distribution network. State estimation can process the measured data and get more accurate state quantity, so that the evaluation of distribution network can be more accurate. The equation of measurement and state in state estimation is;

$$
\mathrm{z}=\mathrm{h}(\mathrm{x})+\mathrm{v}
$$

$\mathrm{z}$ is the measurement vector. $\mathrm{z}=[\mathrm{V} ; \mathrm{P} ; \mathrm{Q}], \mathrm{V}$ is voltage amplitude, $\mathrm{P}$ and $\mathrm{Q}$ are all active and reactive power including real-time measurement data, zero power node, virtual measurement, $\mathrm{x}$ is state vector, $\mathrm{x}=\left[\mathrm{V}_{1}, \mathrm{~V}_{2}, \cdots \mathrm{V}_{\mathrm{N}}, \varphi_{1}, \varphi_{2}, \cdots \varphi_{\mathrm{N}}\right]^{\mathrm{T}}$.

It includes the voltage amplitudes of all nodes and the voltage phase angles except for the balanced nodes. $\mathrm{h}(\mathrm{x})$ is Nonlinear measurement equation. $\mathrm{v}$ is measurement error. Given measurement vector $\mathrm{z}$, state estimation vector $\mathrm{x}$ is an $\mathrm{x}$ value that minimizes the objective function. The objective function is

$$
\mathrm{J}(\mathrm{x})=[\mathrm{z}-\mathrm{h}(\mathrm{x})]^{\mathrm{T}} \mathrm{R}^{-1}[\mathrm{z}-\mathrm{h}(\mathrm{x})]
$$

Because $\mathrm{h}(\mathrm{x})$ is usually a nonlinear function of $\mathrm{x}, \mathrm{x}$ is difficult to calculate directly, the equation can be linearized by Newton's method and then solved iteratively. The derived equation is

$$
\begin{gathered}
\Delta \hat{\mathrm{X}}^{(\mathrm{i})}=\left(\mathrm{H}^{\mathrm{T}}\left(\hat{\mathrm{X}}^{(\mathrm{i})}\right) \mathrm{R}^{-1} \mathrm{H}\left(\hat{\mathrm{X}}^{(\mathrm{i})}\right)\right)^{-1} \mathrm{H}^{\mathrm{T}}\left(\hat{\mathrm{x}}^{(\mathrm{i})}\right) \mathrm{R}^{-1}\left(\mathrm{z}-\mathrm{h}\left(\hat{\mathrm{x}}^{(\mathrm{i})}\right)\right) \\
\Delta \hat{\mathrm{x}}^{(\mathrm{i}+1)}=\Delta \hat{\mathrm{x}}^{(\mathrm{i})}+\hat{\mathrm{X}}^{(\mathrm{i})}
\end{gathered}
$$

Make an iterative correction according to (8) (9) until the objective function J( $\left(\hat{\mathrm{X}}^{(\mathrm{i})}\right)$ is close to the minimum. 


\subsection{Grey Prediction Model}

The grey model is a formal model of differential equation, it is established by making use of the number of discrete random numbers that is generated into a random number which is weakened by randomness. This model is convenient to explore and describe the process of its change. In this paper, the grey prediction algorithm is used to predict the distribution network index by using the advantage of less data needed for forecasting. The specific steps are as follows:

Step1: Make the raw data $x^{(0)}=\left(x^{(0)}(1), x^{(0)}(2), \cdots x^{(0)}(n)\right)$ accumulate through the calculation $\mathrm{x}^{(1)}(\mathrm{k})=\sum_{\mathrm{i}=1}^{\mathrm{k}} \mathrm{x}^{(0)}(\mathrm{i})$, then we can get a series of accumulative numbers $\mathrm{x}^{(1)}=$ $\left(\mathrm{x}^{(1)}(1), \mathrm{x}^{(1)}(2), \cdots \mathrm{x}^{(1)}(\mathrm{n})\right)$;

Step2: Through the calculation $z^{(1)}(k)=\alpha x^{(0)}(k)+(1-\alpha) x^{(0)}(k-1), k=2,3, \ldots, n$, the weighted adjacent value generating sequence is gained. Usually, $\alpha=0$. 5;

Step3: Substitute $\mathrm{k}$ at different times into the grey differentia equation $d(k)+a z^{(1)}(k)=b$, then estimate the value of parameters $a$ and $b$ by least Square method;

Step4: Solute the continuous whitening differential equation $\frac{d x^{(1)}(t)}{d t}+a x^{(1)}(t)=b$, so we can get the predicted value of $\mathrm{x}^{(1)}(\mathrm{k}), \hat{x}^{(1)}(k+1)=\left(x^{(0)}(1)-\frac{b}{a}\right) e^{-a k}+\frac{b}{a}, k=1,2, \ldots, n-$ 1 , then $\hat{x}^{(0)}(k+1)=\hat{x}^{(1)}(k+1)-\hat{x}^{(1)}(k)$.

\section{CASE STUDY}

\subsection{Simulation in the Real-time State}

In this paper, the ieee14 bus network is used as an example. The power reference is 100MVA.The results are the standard value. The upper voltage amplitude of each node is 1.06, the lower limit is 0.94 . There are five distributed power sources with a total generating capacity of 772.4 MW. The actual total active power emitted is 272.4WM. Each measurement data is provided by calculation examples. The wiring diagram of the 14-bus distribution network is as follows.

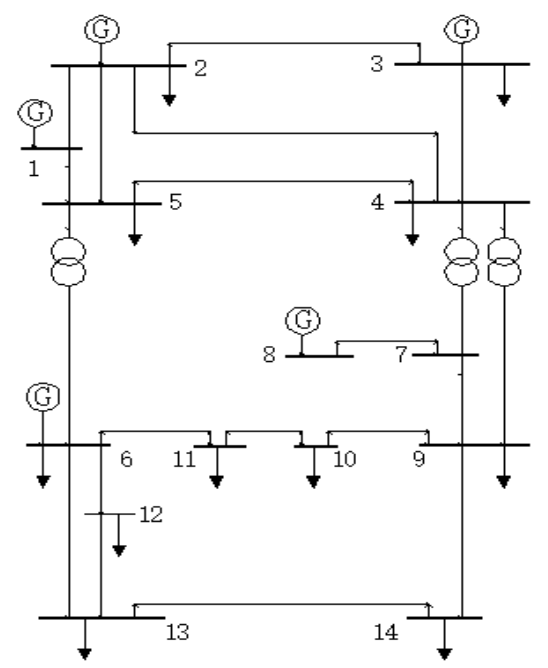

Figure 1. 14-bus distribution network wiring diagram.

And use matlabr2014b to calculate the state on the computer whose CPU is Intel core i5-4210u. This paper does not identify and process the bad data. It is considered that the measured data is not bad data, and the result of the state estimation is as shown in Table 1. 
Table 1. Amplitude and phase angle of each bus voltage

\begin{tabular}{ccc|ccc}
\hline $\begin{array}{c}\text { Bus } \\
\text { number }\end{array}$ & $\begin{array}{c}\text { Voltage } \\
\text { amplitude }\end{array}$ & $\begin{array}{c}\text { Voltage } \\
\text { phase }\end{array}$ & $\begin{array}{c}\text { Bus } \\
\text { number }\end{array}$ & $\begin{array}{c}\text { Voltage } \\
\text { amplitude }\end{array}$ & $\begin{array}{c}\text { Voltage } \\
\text { phase }\end{array}$ \\
\hline 1 & 1.060 & 0.000 & 8 & 1.000 & -13.190 \\
2 & 1.044 & -4.963 & 9 & 1.057 & -14.698 \\
3 & 1.007 & -12.703 & 10 & 1.000 & -13.745 \\
4 & 1.015 & -10.269 & 11 & 1.097 & -14.473 \\
5 & 1.018 & -8.719 & 12 & 1.104 & -14.852 \\
6 & 1.120 & -14.115 & 13 & 1.098 & -14.905 \\
7 & 1.071 & -13.190 & 14 & 1.000 & -14.858 \\
\hline
\end{tabular}

Finally, the security index of distribution network is calculated according to the state estimation of each state quantity, the results are shown in Table 2. By analyzing the existing literature, we can know the optimal range of load margin is $50-60 \%$; the optimal range of voltage margin is $0-7 \%$; the optimal range of load transfer capacity is between $40 \%$ and $50 \%$.The results shows that the network is very flexible and has better load carrying capacity. The voltage of the network is stable. By calculating the current of each line, we know that the load ratio of four lines is between $20 \%$ and $50 \%$, their number is $3-4,2-5,10-11,13-14$. It is concluded that the security of the distribution network is better.

Table 2. Security index of the distribution network

\begin{tabular}{cl}
\hline \multicolumn{1}{c}{ Index } & Value \\
\hline Load margin & 0.664 \\
voltage margin & 0.06 \\
Load transfer capacity & 0.537 \\
Line light load rate & $20 \%$ \\
\hline
\end{tabular}

\subsection{Simulation in the Future State}

In this paper, the data of voltage deviation in one day of a power station are used to simulate the grey model prediction. The program of grey prediction is written by matlabr2014b. The time interval of adjacent data is $1 \mathrm{~min}$. the program uses the voltage deviation of the first 8 times to predict the value of voltage deviation at the next moment, and the Fig.2 is to compare the predicted value of 30 points with the actual value. The blue line represents the predicted value, and the red line represents the actual value. The Fig. 3 is the absolute value of the error between the actual value and the prediction value.

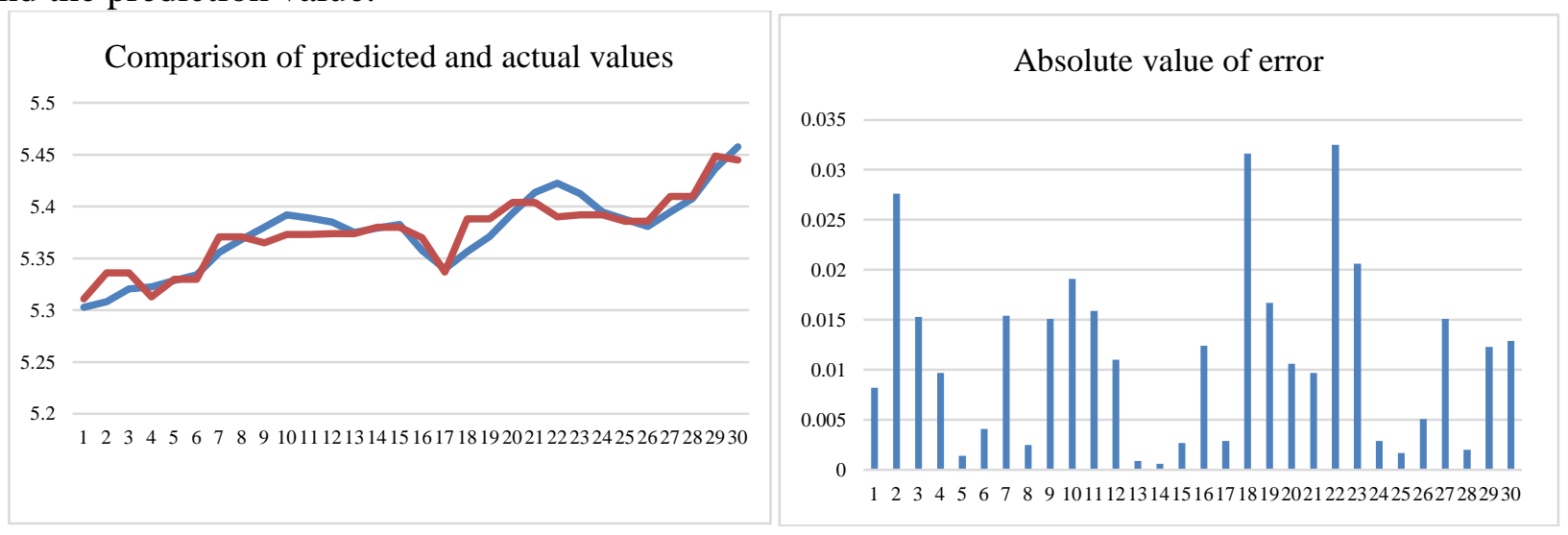

Figure 2. Comparison of predicted and actual values

Figure 3. Absolute value of error 
The prediction effect of the grey prediction model is ideal, it basically conforms to the trend of change, and the error is small. It can be used to predict the future situation by its predicted value, and evaluate the running state of the distribution network in the future state, which is convenient for the operators to make the decision in the next step.

\section{Conclusion}

This paper presents a security assessment method for distribution network based on situation awareness. In the first part, through comprehensive comparison and analysis, the index of better real-time performance has been screened out. In the second part, the state estimation of distribution network is selected as the key to understanding the situation, and the grey model prediction algorithm is selected as the situation prediction algorithm. The key point of this paper is to provide an idea of real-time security evaluation combined with these algorithms. And the simulation results show that the method proposed in this paper can better perceive the real-time security of distribution network and predict the future trend, which can help operators make a better decision.

\section{References}

[1] Shouxiang Wang, Dong Liang, Leijiao Ge .Key technologies of situation awareness and orientation for smart distribution systems ,J. Automation of Electric Power Systems,2016,40(12):2-8.

[2] Zhengrong Wu, Xiaoyong YU, Xuzhu Dong, et al. Real-time situation awareness and evaluation of distribution network based on state estimation ,J. Proceedings of the CSU-EPSA,2018,30(3):140-145.

[3] Manyun Huang, Zhongnong Wei, Guoqiang Sun, et al. A novel situation awareness approach based on historical data-mining model in distribution networks, J. Power System Technology, 2017, 41(4):1139-1142.

[4] Wei Liu, Zhizhong Guo. Research on security indices of distribution networks, J. Proceedings of the CSEE, 2003, 23(8):85-90.

[5] Hongyu Wang, Qinglin Liu, Jiang Bian, et al. Research on safety evaluation method of urban distribution network, J. Electrical application, 2014, 33(2):18-21.

[6] Zejun Ding, Ping Liu, Yangsen Ou. Mechanism of power quality forecast and early warning and their application, J. Proceedings of the CSU-EPSA, 2015, 27(10):27-35. 